\title{
Grasp Frequency and Usage in Daily Household and Machine Shop Tasks
}

\author{
Ian M. Bullock, Student Member, IEEE, Joshua Z. Zheng, Sara De La Rosa, \\ Charlotte Guertler, and Aaron M. Dollar, Member, IEEE
}

\begin{abstract}
In this paper, we present results from a study of prehensile human hand use during the daily work activities of four subjects: two housekeepers and two machinists. Subjects wore a head-mounted camera that recorded their hand usage during their daily work activities in their typical place of work. For each subject, 7.45 hours of video was analyzed, recording the type of grasp being used and its duration. From this data, we extracted overall grasp frequency, duration distributions for each grasp, and common transitions between grasps. The results show that for 80 percent of the study duration the housekeepers used just five grasps and the machinists used 10. The grasping patterns for the different subjects were compared, and the overall top 10 grasps are discussed in detail. The results of this study not only lend insight into how people use their hands during daily tasks, but can also inform the design of effective robotic and prosthetic hands.
\end{abstract}

Index Terms-Human grasping, manipulation, robotic hands, prosthetics, activities of daily living

\section{INTRODUCTION}

U NDERSTANDING how humans utilize their hands has long been a topic of interest. Initially, this interest was primarily related to applications such as biomechanics, hand surgery, and rehabilitation [1], [2], [3], [4]. With the advent of robotics into manufacturing tasks, the study of hand function received new life as researchers began to investigate human hands to shed light on the design and control of robotic end effectors.

This study presents an investigation into grasp type and frequency for common classes of manipulation tasks. In particular, we investigate the hand use behavior of two fulltime housekeepers and two professional machinists-two areas of interest for robotic assistants. Subjects wore a small nonobstructive head-mounted camera with a wide-angle lens to film their hand workspace during work-related activities. For each subject, 7.45 hours of video was analyzed, spanning a wide range of tasks representative of their profession.

Previous grasp studies have primarily focused on the hand posture used for preselected objects, as opposed to recording unstructured human manipulation behaviors. An early study related to prosthetics [3] photographed 12 subjects to determine hand prehension shapes used in picking up 27 objects and the "hold-for-use" posture for 57 objects. Santello et al. [5] asked subjects to imagine

- I.M. Bullock, S. De La Rosa, C. Guertler, and A.M. Dollar are with the Department of Mechanical Engineering and Materials Science, Yale University, 9 Hillhouse Ave., Dollar Lab, New Haven, CT 06511.

E-mail: \{ian.bullock, sara.delarosa, charlotte.guertler, aaron.dollar\}@yale.edu.

- J.Z. Zheng was with the Institute for Physical Sciences (IPS), Mclean, VA 22101-3616. E-mail: josh.z.zheng@gmail.com.

Manuscript received 2 Aug. 2012; revised 18 Dec. 2012; accepted 15 Feb. 2013; published online 21 Feb. 2013.

Recommended for acceptance by A. Okamura.

For information on obtaining reprints of this article, please send e-mail to: toh@computer.org, and reference IEEECS Log Number TH-2012-08-0059.

Digital Object Identifier no. 10.1109/TOH.2013.6. grasping 57 test objects while a motion capture system recorded 15 finger joint angles. Through principal components analysis on the kinematic hand movements, the results showed that the first two components could account for $\sim 80$ percent of the variance. Finally, Sperling and Jacobson-Sollerman [6] coded general hand surfaces and grasp types used for 30 subjects during video of a standardized meal eating task, giving data for 1,277 total coded grips.

As described in detail in Section 2, Cutkosky [7] studied the grasps utilized by machinists using single-handed operations in working with metal parts and hand tools. Kemp created a wearable system including a head-mounted camera and orientation sensors mounted on the body to learn body kinematics (not including the hand) and record manipulation tasks. A large amount of manipulation video was recorded but was never analyzed for details of grasp and object type [8]. While these previous efforts have helped better understand human grasp behavior, none have formally recorded and evaluated grasp type and frequency over a large time span of daily use.

For robotic and prosthetic applications, there are a number of reasons why the human hand should not or cannot be simply copied to produce effective end effectors and terminal devices. With its 21 controllable degrees of freedom, 38 muscles, and thousands of sensory organs, the human hand is incredibly complex. Current state of the art in engineered systems simply cannot achieve that level of complexity and performance in the same size package. Furthermore, with added complexity comes added cost and lower durability. However, very few, if any, practical grasping and manipulation tasks for robotics or prosthetics require the full complexity of the human hand. Indeed, as evidenced by the widespread use of simple prosthetic terminal devices such as the single DOF split hook [9], even the simplest devices, if well designed, can have a great deal of utility. 
The benefits of lower-complexity devices have not been overlooked in the robotics and prosthetics research communities. A number of simplified hands have been developed, many of which are underactuated to provide passive adaptability and, in turn, a larger range of grasp configurations per actuator, (e.g., [10], [11], [12], [13], [14]). The design of prosthetic hands comes with additional challenges related to the limited amount of space and weight that can be implemented, particularly in light of the fact that amputations can be performed at various points on the limb, limiting most devices to the space distal to the wrist (e.g., [15], [16]).

Due to the many reasons why the full spectrum of human hand capabilities cannot be practically achieved, some smaller subset of those must be chosen. We expect this study to help to motivate that choice by informing which human grasp types are most commonly used for household and other important tasks. For example, a robotic or prosthetic hand could be designed to achieve the five most frequent grasps, rather than trying to implement every possible human grasp type, to reduce the hand complexity while maintaining much of the functionality.

We begin this paper with a discussion of human grasp classification, presenting an overview of previous work and laying out the terminology used in this paper. We then describe our experimental methodology, including details on the subjects used, apparatus, and protocol. We then present the results for the four subjects, two professional housekeepers and two professional machinists, identifying the frequency of grasp type use for each. Finally, we discuss limitations of the current study and future work.

\section{Human Grasp Classification}

Schlesinger [2] made the first major attempt to organize human grasping behavior into distinct categories: cylindrical, tip, hook, palmar, spherical, and lateral. These grasps are primarily defined by the object that the hand interacts with. However, human grasp choice is also determined by the tasks that need to be accomplished. In 1956, Napier [1] suggested a scheme that would divide grasps into power and precision grasps. Unfortunately, not all the grasps fell cleanly into either of these two categories, with intermediate grasps such as the lateral pinch serving both power and precision functions.

In studying the grasps required for manufacturing tasks, Cutkosky [7] provided a much more comprehensive and detailed organization of human grasps. This taxonomy was acquired through a set of observational surveys on professional machinists and integrated the previous work done by Schlesinger and Napier. The Cutkosky taxonomy tree is organized first by power and precision grasps, and then by shape and function down the tree.

In this paper, we utilize a slightly extended version of the comprehensive taxonomy presented by Feix et al. [17]. This taxonomy is the most complete in existence, in the authors' opinion, but lacks nonprehensile grasps, which we have added, specifically the platform grasp ("platform") from Cutkosky's taxonomy [7]. We define a grasp by the Feix definition as "every static posture with which an object can be held securely with one hand," but drop the final "irrespective of the hand orientation" which is in the original definition. Nonprehensile activities such as pressing a button are not included. We also kept the original Cutkosky naming for the grasps, such as using thumb-n finger instead of the Feix taxonomy's "prismatic n finger." Feix's "adduction grip" is simply called "adduction." The final taxonomy used in this paper is given in Fig. 1. All of Feix's grasps were observed in the data set except for the distal type, a specialized grasp for scissors. Note that no grasps were seen that did not fall into this taxonomy, but some license was taken to fit some into it, especially with soft objects such as towels (as discussed further in Section 5.3).

Though there have been a number of efforts focused on classifying types of human grasps, the authors are not aware of any studies examining grasp frequency in a reallife setting, aside from the preliminary conference paper preceding the current work [18]. A related study looks at the instances that certain high-level manipulation tasks are used [19], but uses a very broad classification and a fairly small data set. Frequency data are important as it will prioritize grasp types according to the most frequently used in the examined daily activities and can serve to help motivate design choices in robotic and prosthetic hands, helping the designer to address tradeoffs between complexity and performance.

\section{Methods}

Two machinists and two housekeepers participated in the study discussed in this paper. The first machinist (machinist 1 ) is a 41-year-old right-handed male with more than 20 years of professional machining experience, and the second machinist (machinist 2) is a 50-year-old righthanded male with about 30 years of experience. The first housekeeper (housekeeper 1) is a 30-year-old right-handed female with five years of housekeeping experience, and the second housekeeper (housekeeper 2) is a 20-year-old righthanded female with eight months of experience. None of the four subjects had any history of injury or any disability that would affect grasping and manipulation behavior. Machinist 2 did report prior shoulder injury due to repetitive overhead reaching, but did not experience any issues during the duration of the study.

The following enrollment criteria were used to screen potential subjects for the study: significant experience as professionals in their field, of normal physical ability, right handed, able to participate to the extent to generate 8 hours of useful data, and performing a wide variety of tasks representative of their profession during the span of their participation. For example, one machinist subject who almost entirely used a CNC lathe was excluded in favor of another subject who performed a wider variety of manual machining tasks on several different machines. Subjects were paid $\$ 10$ per hour for participation on top of their normal salary.

\subsection{Experimental Procedure and Apparatus}

Subjects meeting the enrollment criteria above were enrolled in the study and provided instruction regarding the protocol. The subjects wore the head-mounted camera shown in Fig. 2 (top and middle). The wire from the 


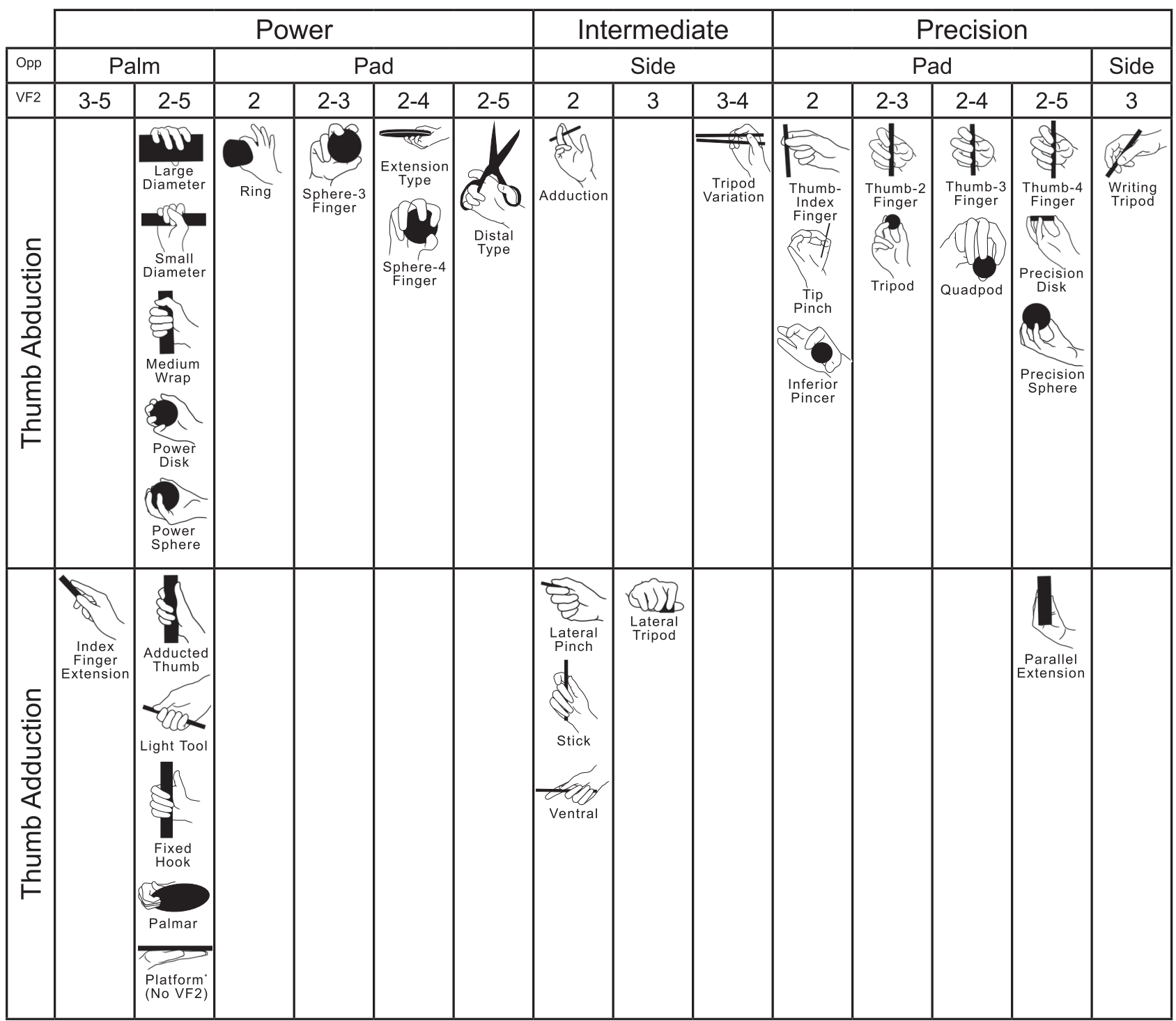

Fig. 1. Modified Feix grasp taxonomy [17]. Opp refers to the opposition type used in the grasp, while VF2 is the second virtual finger used in the grasp. Note that a few names are used from the Cutkosky taxonomy, such as for the thumb-n finger grasps. The platform grasp from the Cutkosky taxonomy is shown, although it does not have a second virtual finger.

camera is routed to the recorder on the back of the subject over the top of the head to the waist. Subjects were instructed on how to start and stop the recording when privacy was required.

A total of at least 8 hours of hand usage was recorded for each subject, over multiple days. We discussed with the subjects their overall work responsibilities to confirm that the data we had taken was representative of the general set of tasks that they perform for their profession. The total data recording process took place over 3-5 days of work for each subject, in some cases resulting in more than the 7.45 hours of video analyzed for this study.

The hardware, shown in Fig. 2, consists of a tube camera (RageCams, model 3225, $200 \mathrm{~g}$, 22-mm dia $\times$ 60-mm long, $640 \times 480$ resolution) with a wide-angle fisheye lens ( $2.5 \mathrm{~mm}, \sim 140$ degrees field of view) attached to a threeband head strap taken from a hiker's lamp. This setup allows the camera to rest on the subject's head without being intrusive or uncomfortable. The camera is connected to a mini digital video recorder (AngelEye 2.4-GHz PVR,
$115 \times 65 \times 25 \mathrm{~mm}, 30$ FPS) with approximately 2 hours of recording time on a single battery charge, stored on a 16-GB SD card. An external battery pack $(12 \mathrm{~V}, 8 \times \mathrm{AA})$ is used to power the camera. Both the receiver and battery pack were placed in the back pocket of the subject.

The setup is able to acquire video of sufficient quality for manual grasp classification. The overhead view was chosen after informal testing showed this to be the most useful for our purposes as it shows the entire workspace of both left and right arms in front of the body as well as enough of the surroundings to give the context of the grasps in addition to the grasp itself. Fig. 2 (bottom) shows a sample image taken with this setup.

To analyze the video data, researchers who were trained in classifying grasps monitored the slowed-down video. Specifically, two researchers were trained to perform this grasp analysis. The training first involved extensive literature review in the area of human grasping, followed by reading a formal set of guidelines, which describe how to apply the modified Feix taxonomy (Fig. 1) to tagging the 

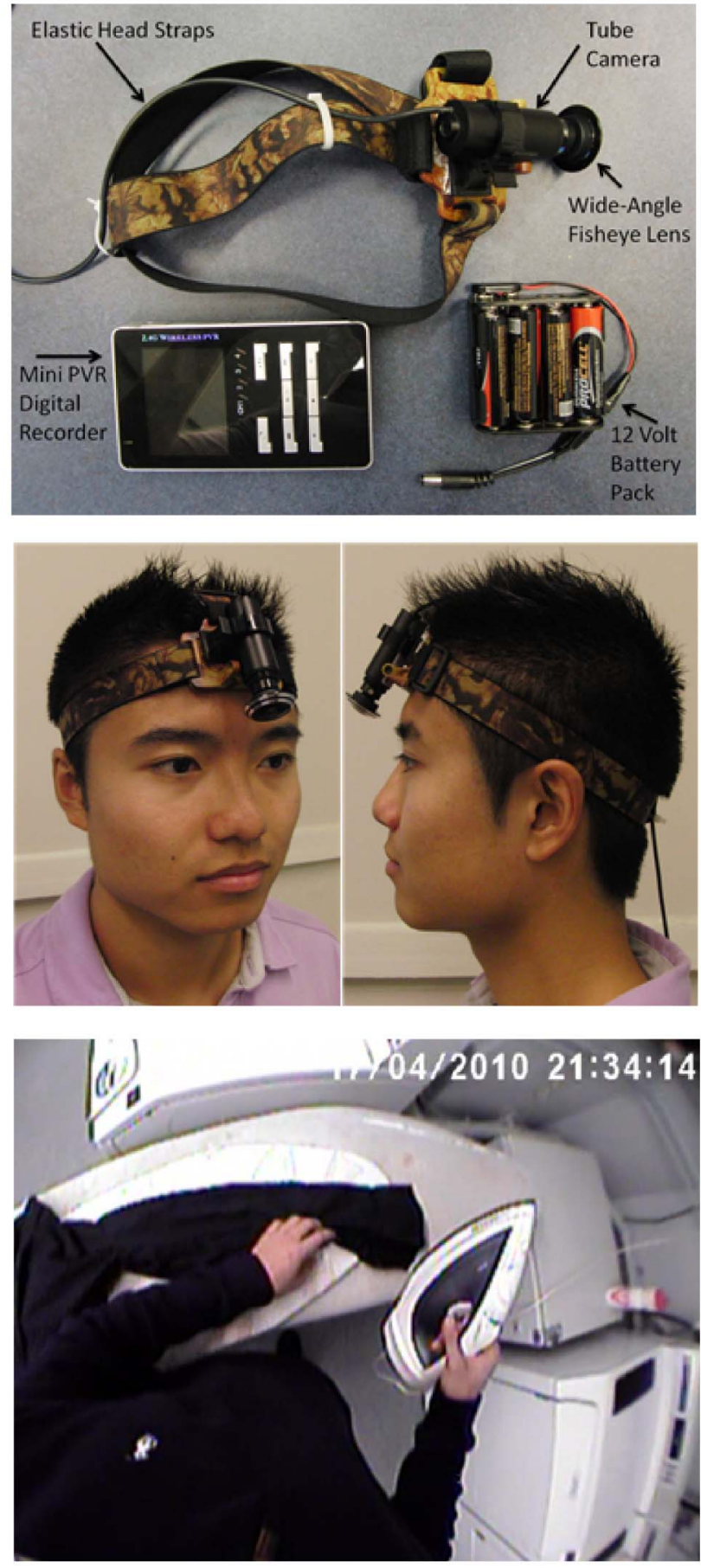

Fig. 2. Camera and receiver hardware (top), example of the apparatus as worn by a subject (middle), and sample image from the system in use.

video data. During the rating process, researchers referred to an alphabetically sorted visual chart of the grasps, to make it easy for them to remember any uncommon grasps. This was used to help reduce any biases toward common grasps that may be easier to remember.

The coding guidelines were such that whenever the subject changes their grasp, the new grasp is recorded, along with the time stamp at which the switch was made. Quick grasp transitions lasting less than a second are not recorded. Additionally, the object that the subject was grasping as well as what they were doing with the object is recorded. Only the right (dominant) hand was examined in the present study. In cases of occlusion, the continuous nature of the video generally allowed the raters to make a very good guess at the grasp (i.e., usually there are not sudden changes in hand configuration during brief moments, where the hand is obscured). In extreme cases, the raters did occasionally mark grasps as "unknown."

Each video segment was tagged by one of the two researchers. It was decided to use a single rater per segment to allow much more video data to be analyzed in a reasonable time frame, as well as due to the extensive training required for each rater. The statistical simulation methods described in Section 4.1 use information from 2 hours of video rated by both raters to model the additional uncertainty introduced by using a single rater.

A small amount of data cleanup was required following these initial data tagging efforts. Due to a cable reliability issue during the housekeeper 1 data recording, some data points were removed, reducing the final data duration to 7.45 hours. Since the cable issue caused the image to go completely black rather than partially affecting image quality, no systematic errors should be introduced into the data from this incident. The data from the other subjects were then trimmed down to the 7.45-hour duration from housekeeper 1 to match the subject data length.

The other main cleanup step was to handle a few instances where multiple grasps were recorded by the raters, usually when the subject was carrying multiple objects with their dominant hand. While this could be an interesting area of future study, for the present work, the principal, first grasp is taken if multiple simultaneous grasps were recorded.

\subsection{Inter-Rater Agreement}

Since two raters were used to analyze the video used in this study (approximately 50 percent of the data per rater), an inter-rater reliability assessment was performed using a modified Cohen's $\kappa$ method [20]. Since there were not discrete questions involved in classifying grasps, the confusion matrix was created by recording the durations of agreement or disagreement in the tagged grasp over the same sample of data, as suggested in [21]. Two 1-hour samples of data were prepared from several different videos, 1 hour from the machinists and 1 hour from the housekeepers. While the samples were mainly taken from two of the subjects (housekeeper 1 and machinist 1), the types of grasps in the sample set should still be representative of the four subjects, because very similar tasks were being performed by the pairs of subjects in each profession.

The 1-hour housekeeper sample was rated at the beginning of the study, while the machinist sample was rated after completion of the study. Thus, the housekeeper sample can be seen as a best case view of the rater reliability, while the machinist sample is a worst case view, because ratings can drift over the course of a study. Because of this, we have opted to average the two samples to produce an overall confusion matrix. While there may be some slight differences between the characteristics of the grasps in the machinist and housekeeper data, the grasp classification task should be fundamentally similar enough across professions to make this combination acceptable. The 


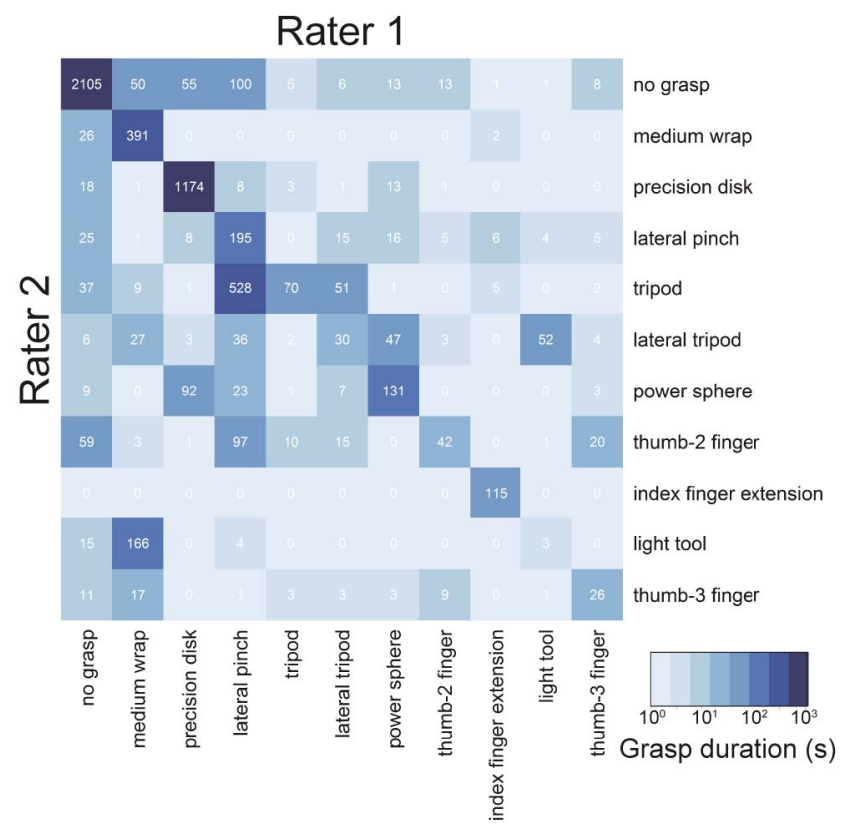

Fig. 3. Confusion matrix to evaluate inter-rater reliability for the two raters used. Note that the colors are plotted on a logarithmic scale that emphasizes the elements off of the main diagonal more than a linear scale would.

portion of the resulting confusion matrix representing the 10 most frequent grasps can be seen in Fig. 3 .

Cohen's $\kappa$ was then calculated using this confusion matrix, giving $\kappa=0.54$. This represents the proportion of agreement that is not due to chance. Some of the disagreement can be explained by different timing in the recording of the start and stop of the grasps. For example, the "no grasp" category has an agreement of about 90 percent, suggesting that the start and end of the grasp were recorded at a slightly different point in the video by the two raters. This type of timing disagreement should only produce random error and not systematic error over the course of the full study.

Some of confusions of apparently dissimilar grasps can also be explained by rater timing differences. For example, for the unusual confusion of power sphere and lateral pinch, a matrix of grasp transitions (Fig. 8) shows lateral pinch to power sphere and vice versa are both common transitions. For a few other grasps, there may be actual classification disagreement rather than timing disagreement.

A few grasps appear to be harder to reliably distinguish, and produced disagreement between the raters. Lateral grasps such as lateral pinch and lateral tripod appear to produce disagreement. One possible reason for this is that some hand positions might be in between a lateral grasp configuration and a nonlateral precision grip such as a tripod, so the classification is unclear. In other cases, motions such as the knob turning frequently performed by the machinist may involve dynamic transitions between more or less lateral configurations. Another gray area may occur when the object is not clearly in the fingertips or in the hand, but somewhere in between. This could help to explain the confusion between the precision disk and power sphere grasps. Finally, the medium wrap and light tool grasps are frequently confused by the two raters.

Overall, $\kappa=0.54$ shows a high amount of inter-rater agreement, and most of the disagreement appears to be due to timing of the grasp transitions, as evidenced by the confusion matrix being fairly symmetric. We, thus, proceeded with further analysis of the video.

\section{Results}

For the 7.45 hours of video for each subject, a surprisingly large number of grasps and grasp transitions were seenapproximately 4,700 per subject. Four sample images for each of the top 10 grasps are presented in Fig. 4. We first present the frequency data (Section 4.1), then compare the

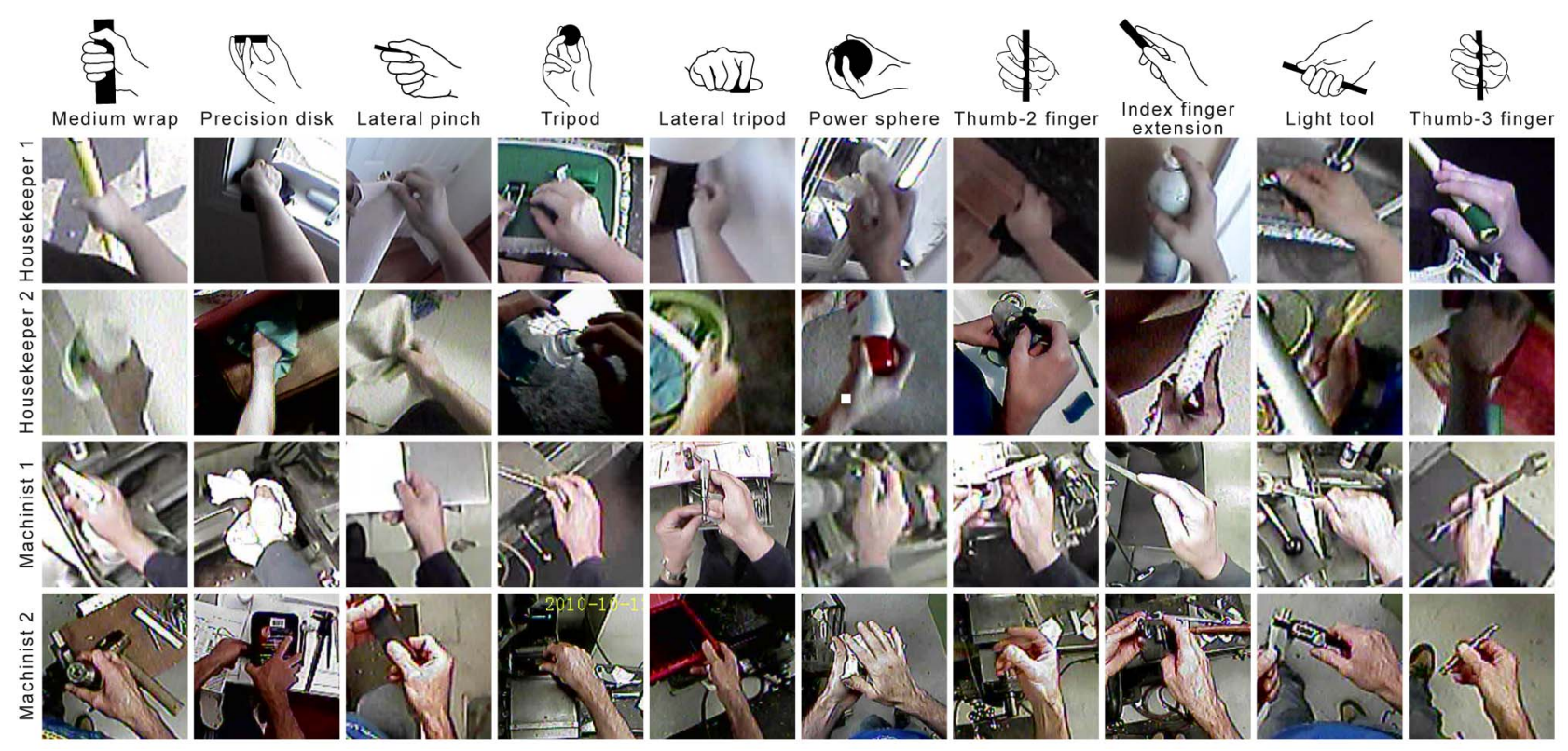

Fig. 4. Example images for each of the top 10 grasps from all four subjects. Note that some grasps do not fit the model image perfectly; for example, the precision disk was often used for wiping motions. 


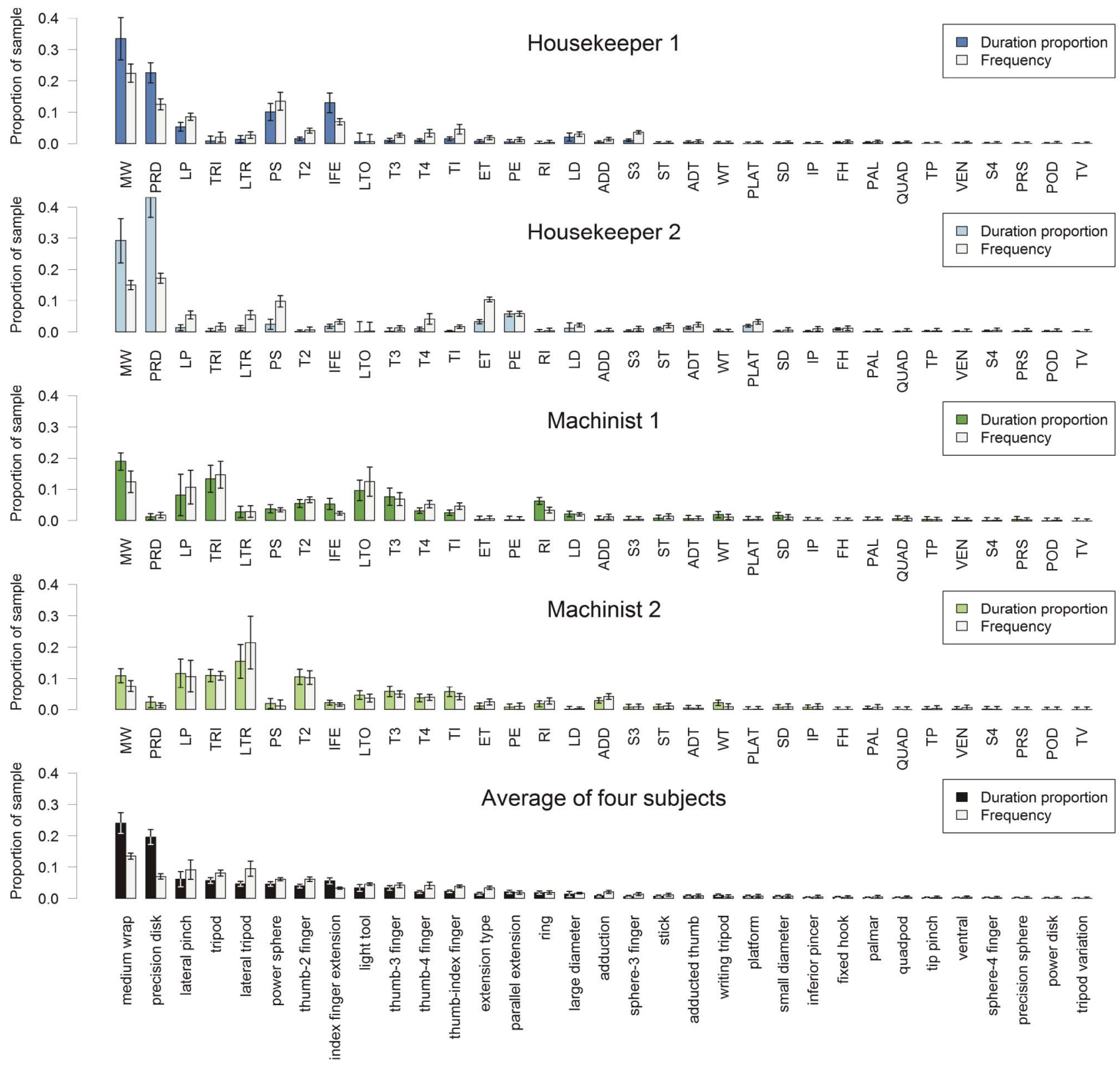

Fig. 5. Frequency and duration proportion of grasps for each of the four subjects as well as for the four subjects combined. Duration proportion is calculated by the duration for a grasp divided by total duration of all grasps, and frequency (counts) is calculated by the number of instances of the grasp divided by the total number of grasp instances for that subject.

two subjects of each class (Sections 4.2 and 4.3), and then between classes (Section 4.4). Next, we present the duration distributions for how long each grasp was held (Section 4.5), and finally common transitions between grasps (Section 4.6).

\subsection{Summary of Results}

The calculated grasp frequencies and duration proportions for each grasp recorded for the four subjects can be seen in Fig. 5. Duration proportion is calculated by the total duration of a given grasp type divided by the total duration of all grasp types, and frequency is calculated by the number of instances of a grasp divided by the total instances of all grasps. The frequency and duration proportions are provided for all four subjects, with a plot of the averages at the bottom. The grasps are sorted in order of a decreasing average of the duration proportion and frequency for each grasp.

Error bars are estimated using a case resampling bootstrap method (see, e.g., [22]), modified to incorporate the confusion matrix data. Specifically, for each subject, samples are drawn randomly from the original data set (with replacement) to match the length of the original data. Then, a symmetric, normalized version of the confusion matrix is used to introduce random transitions from the originally sampled grasp to what another rater would have specified. These random transitions are introduced for a given grasp data point with probability $p$ chosen from the uniform distribution $U(0,1)$.

After this sampling procedure is completed, grasp frequencies for the sample are calculated. This sampling is repeated $N=1,000$ times, with a new probability $p$ of 
TABLE 1

Top 10 Grasps with Averages for the Four Subjects

\begin{tabular}{|c|c|c|c|}
\hline Grasp & $\begin{array}{l}\text { Duration } \\
\text { Proportion }\end{array}$ & Frequency & $\begin{array}{l}\text { Mean } \\
\text { Duration } \\
\text { Per Grasp }\end{array}$ \\
\hline medium wrap & $23 \pm 2 \%$ & $14.0 \pm 0.5 \%$ & $12 \mathrm{~s}$ \\
\hline precision disk & $17 \pm 1 \%$ & $8.2 \pm 0.4 \%$ & $19 \mathrm{~s}$ \\
\hline lateral pinch & $7 \pm 1 \%$ & $9 \pm 2 \%$ & $4.5 \mathrm{~s}$ \\
\hline tripod & $6.4 \pm 0.5 \%$ & $7.4 \pm 0.5 \%$ & $4.8 \mathrm{~s}$ \\
\hline lateral tripod & $5.3 \pm 0.4 \%$ & $8 \pm 1 \%$ & $3.3 \mathrm{~s}$ \\
\hline power sphere & $4.6 \pm 0.4 \%$ & $7.0 \pm 0.3 \%$ & $5.1 \mathrm{~s}$ \\
\hline thumb-2 finger & $4.5 \pm 0.3 \%$ & $5.5 \pm 0.4 \%$ & $4.3 \mathrm{~s}$ \\
\hline $\begin{array}{l}\text { index finger } \\
\text { extension }\end{array}$ & $5.6 \pm 0.5 \%$ & $3.6 \pm 0.2 \%$ & $11 \mathrm{~s}$ \\
\hline light tool & $3.7 \pm 0.6 \%$ & $4.3 \pm 0.2 \%$ & $5.0 \mathrm{~s}$ \\
\hline thumb-3 finger & $3.7 \pm 0.4 \%$ & $4.0 \pm 0.4 \%$ & $5.4 \mathrm{~s}$ \\
\hline
\end{tabular}

confusion matrix resampling chosen from $U(0,1)$ each time. This variation in confusion matrix resampling of the simulated data models the effects of rater variability on the resulting frequency estimates. Error bars are plotted for a 95 percent confidence interval based on the standard deviation of the 1,000 different frequency estimates for each grasp. For a few rare grasps, not enough confusion matrix data were available to effectively calculate the standard deviation directly from the simulation. For these grasps, the standard deviation was computed based on interpolating with a linear fit of standard deviation to grasp frequency-in general, the uncertainty of the grasp frequency appears to scale with the frequency itself, although there are some outliers.

When compared to a simple bootstrap resampling simulation without using the confusion matrix data, the standard deviation estimate for the summary four subject data is on average 2.5 and 3.9 times as large for the frequency and duration proportion, respectively. For the top 10 grasps only, the standard deviation is 1.6 and 2.4 times as large for frequency and duration proportion, respectively. Thus, modeling the inter-rater confusion effects increases the uncertainty estimate significantly, especially for the rarer grasps that are generally confused more often.

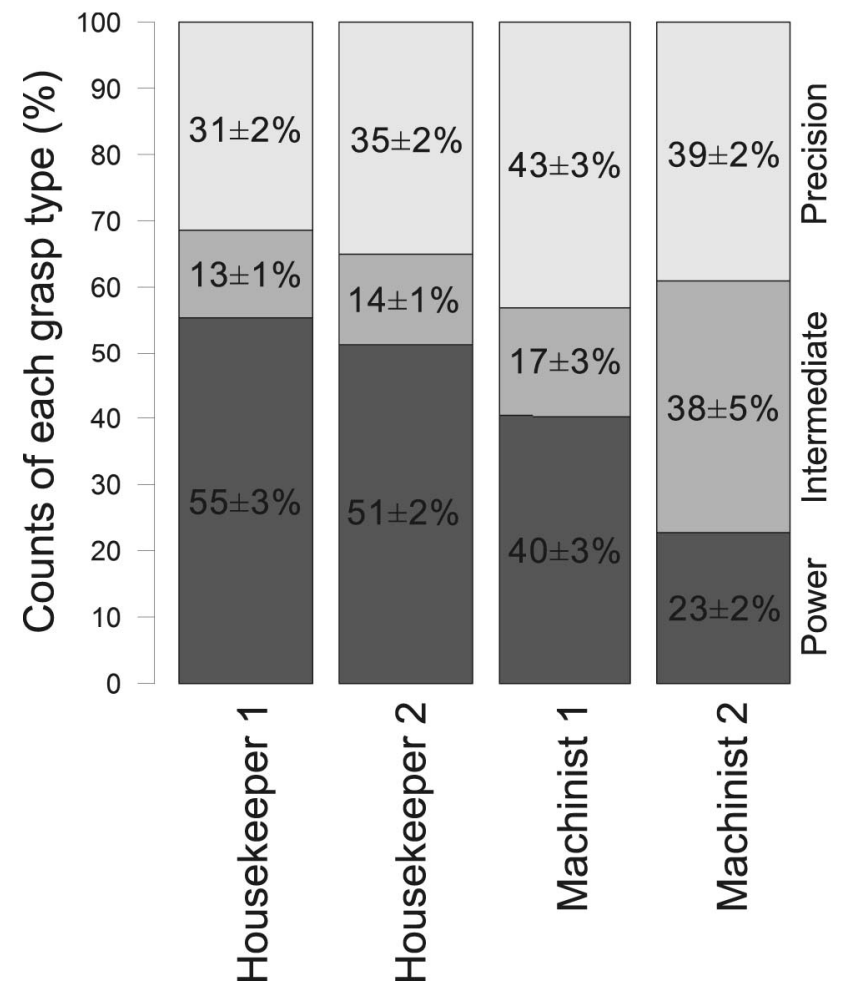

Fig. 6. Power, intermediate, and precision grasps for each of the four subjects. The color for each grasp type is labeled at right. Note that the housekeepers show a higher proportion of power grasps, and machinist 2 shows a high proportion of intermediate grasps largely due to frequent lateral tripod use.

These error estimates appear to be reasonable based on a visual analysis of how the frequency estimates settle as a function of increasing sample size. Note, however, that the resulting confidence intervals only model the error caused by the sample size, grasp distributions, and inter-rater variability, and do not account for other possible sources of experimental error.

Table 1 shows the average durations and frequencies for the top 10 grasps (reported with uncertainties of plus or minus a single standard deviation), which account for approximately 80 percent of the total grasp duration. The other 23 grasp types observed in the data set, therefore, only represent about 20 percent of the observed grasps. Medium wrap and precision disk have very high-duration proportions (23 and 17 percent). Following these two grasps, the average frequencies and duration proportions decrease fairly gradually from lateral pinch to thumb-3 finger, from about 8 percent to about 4 percent of the total sample.

Since this summary plot does not include the no grasp category, the proportion of time spent grasping by each subject should be addressed. Housekeepers 1 and 2 spent 77 and 90 percent of the study duration grasping, respectively. Machinists 1 and 2 were grasping for 69 and 63 percent of the study duration, respectively.

One can also consider the proportion of power, precision, and intermediate grasps used by each subject, according to the Feix classification. This information is displayed in Fig. 6. If the data from all four subjects is combined, the resulting proportion is 40.0 percent power grasps, 22.4 percent intermediate grasps, and 37.6 percent precision grasps, 


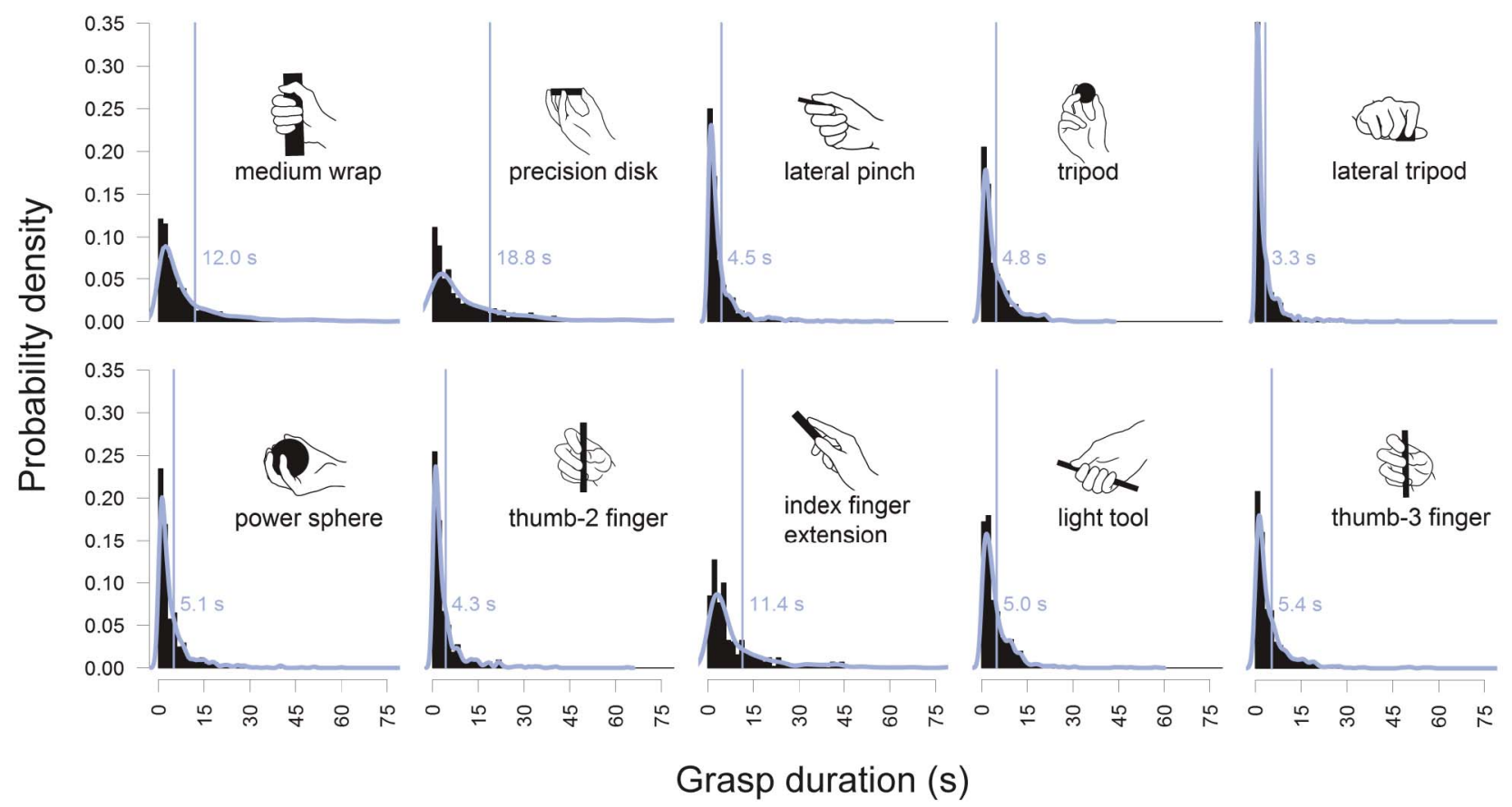

Fig. 7. Grasp duration histograms for the top 10 grasps. Kernel density curves are fitted for each histogram, and the labeled line corresponds to the mean duration for each grasp.

when calculated by frequency (as opposed to the duration proportion). Note that lateral pinch and lateral tripod are the most common intermediate grasps. Overall, the machinists appear to use fewer power grasps. Machinist 2 uses the largest number of intermediate grasps, mostly due to his increased usage of the lateral tripod grasp.

\subsection{Housekeeper Comparison}

It should be acknowledged first that a part of the intersubject and interprofession differences could be attributed to slightly different proportions of data analyzed by each rater for each subject, but most of this effect should be taken into account by the increased confidence intervals for grasps with frequent inter-rater confusion. Many frequency differences between Housekeepers 1 and 2 are observed. P-values for differences in grasp frequency between the two subjects were calculated using a Z-test based on the standard deviations calculated in the statistical simulations used to produce the confidence intervals. Differences for which the duration proportion difference and frequency difference are both statistically significant will be emphasized. The largest magnitude difference is in the precision disk duration proportion-Housekeeper 2 used the precision disk 43 percent of the time, while Housekeeper 1 used it only 23 percent of the time $(p<0.001)$. Lateral pinch and index finger extension were more common in Housekeeper 1, while extension type and parallel extension were more common in Housekeeper 2 (all $p<0.001$ ). For medium wrap, Housekeeper 1 had higher frequency and duration proportion, but the duration proportion difference is not significant $(p=0.2)$ while the frequency difference is $(p<0.001)$.

\subsection{Machinist Comparison}

The grasp data for the two machinist subjects can be similarly compared and also have many statistically significant differences. Machinist 1 has higher frequency use of the medium wrap and light tool grasps $(19,10$ percent duration, respectively) compared to Machinist 2 (11, 5 percent duration, respectively), with $p<0.001$. The largest magnitude difference is that Machinist 1 uses the lateral tripod grasp much less than Machinist $2(p<0.001)$. Machinist 1 uses the lateral tripod 3 percent of the time by duration and counts whereas Machinist 2 uses the grasp with 16 percent duration and 22 percent frequency.

\subsection{Housekeeper versus Machinist Comparisons}

Overall, the observed variability between subjects of the same profession was unsurprisingly less than the variability between subject types, after averaging the data for the two subjects in each profession. The two largest effects observed are that the machinists used the medium wrap and precision disk grasps significantly less than the housekeepers. Lateral pinch, tripod, lateral tripod, thumb-2 finger, and light tool were all used more by the machinists, while power sphere and index finger extension were used more by the housekeepers.

\subsection{Grasp Durations}

Duration histograms for the top 10 grasps are shown in Fig. 7. The thin vertical lines are at the mean grasp duration for each grasp. It can be easily seen that some grasps, such as the lateral tripod, are mainly used for short durations, while others, such as precision disk, have both a higher mean duration and a much heavier tail distribution. It should be noted that there are 106 samples in the data set that lie beyond the plotted 75 second range, but this accounts for only 0.6 percent of the data set. 


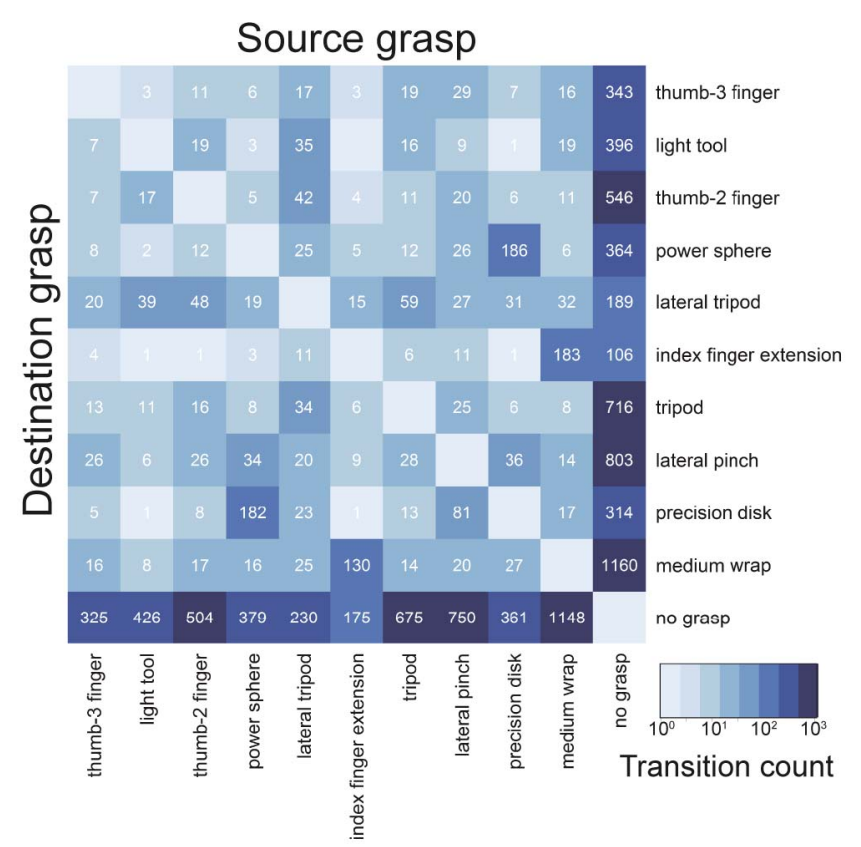

Fig. 8. Grasp transition matrix for the top 10 grasps and the no grasp category. The counts labeled are the number of transitions from a given source grasp (columns) to a given destination grasp (rows).

\subsection{Grasp Transitions}

Note that because raters were instructed only to record grasp transitions lasting more than a second, many short manipulative motions may be missing in the present data set. However, the sequence of grasps in the data set can still be used to understand some of the common, longer duration grasp transitions during object manipulation.

By summing the transitions between different grasps in the entire data set, we can construct a grasp transition matrix $T$, where each element $t_{i j}$ is the total number of transitions from grasp $j$ to grasp $i$. A heat map illustrating the grasp transition matrix for the top 10 grasps can be seen in Fig. 8. A column of this matrix shows the number of different transitions to each grasp from the source grasp corresponding to that column. A row of the matrix shows the number of transitions to the grasp of that row from each source grasp. In general, the grasp transition matrix appears to be fairly symmetric, with a similar number of transitions between a given pair of grasps in either direction. The top 10 most common grasp transitions are illustrated in Fig. 9. Note that the percentages given are for the proportion of overall grasp transitions in the entire data set. The common transitions for the top five grasps will now be discussed briefly.

For the most common grasp, medium wrap, the most transitions are to and from index finger extension, 183 and 130 counts (1.0, 0.7 percent of all transitions), respectively. Medium wrap transitions to and from many other grasps such as precision disk, lateral pinch, and lateral tripod. For precision disk, by far the most transitions are to and from power sphere (186 and 182 counts, 1.0 percent), which may be a common power-precision manipulation transition. Precision disk also frequently follows lateral pinch (81 counts, 0.4 percent), suggesting that a lateral pinch could be used to prepare an object for manipulation in a

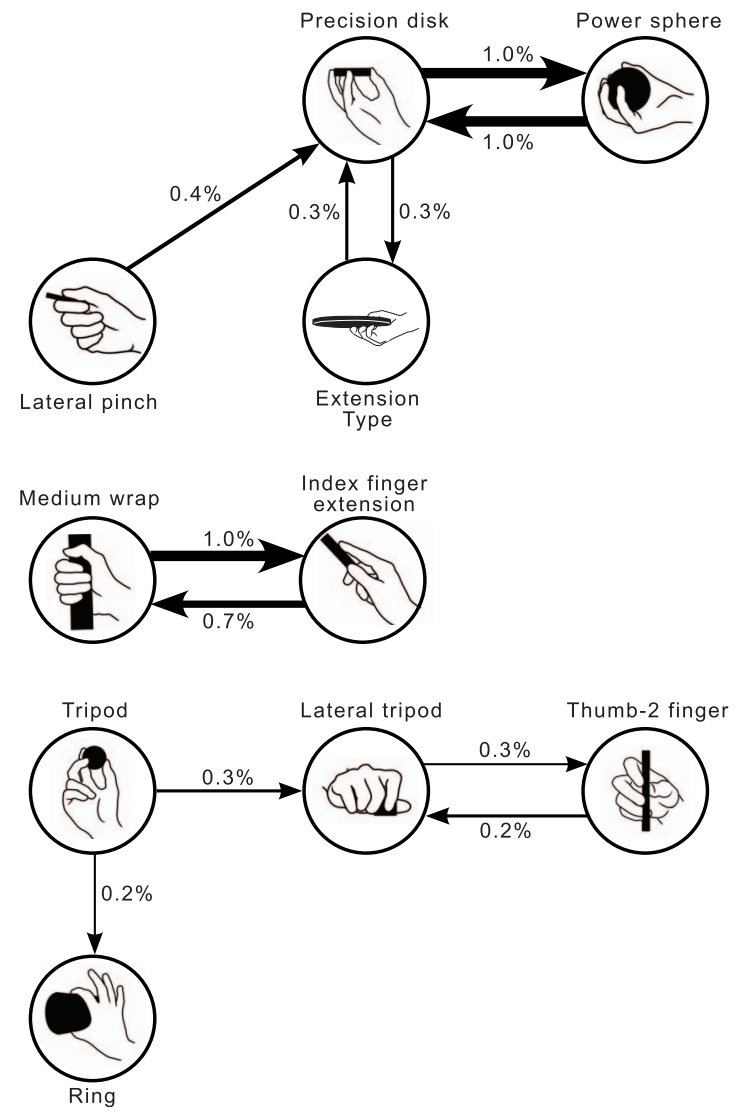

Fig. 9. Top 10 grasp transitions by frequency. Percentages indicate proportion of all grasp transitions in the data set. Arrow thickness also indicates frequency. Note that many other transitions occur in the data between the grasps shown; some have only slightly lower prevalence than the top 10 transitions shown.

precision disk configuration. This transition is the most frequent for the lateral pinch grasp as well. The tripod grasp frequently transitions to and from the lateral tripod (59 and 34 counts, 0.3 and 0.2 percent). Finally, the fifth grasp, index finger extension, most frequently transitions to and from medium wrap (130 and 183 counts, 0.7 and 1.0 percent).

The frequency of the transitions from no grasp to various grasps may show whether the grasp is suitable for initially picking up an object, or whether it is generally transitioned to after the object has already been grasped. Most of the top 10 grasps appear to be frequently used for initial prehension, however there are some exceptions. Precision disk, lateral tripod, and index finger extension are transitioned to from no grasp less than half as much as from another grasp.

In a similar manner, we can consider the frequency of transitions to no grasp to evaluate which grasps are generally used to release objects from the hand. While many of the top 10 grasps often show an object release transition, for a few grasps it is more uncommon. Lateral tripod, index finger extension, and precision disk transition to no grasp $0.64,0.82$, and 0.8 times as much as to another grasp, whereas for many grasps this proportion is greater than 2 .

\section{Discussion}

As mentioned above, a small number of grasps are used for the majority of the time. Specifically, the top 3 grasps 
already account for 50 percent of the data set by duration and 30 percent by frequency. The top 10 grasps account for 81 percent of the duration and 72 percent of the frequency. Covering 95 percent of the study data requires a full 19 grasps for 95 percent duration and 21 for 95 percent frequency. While the most common grasps are similar across all subjects, there is some variability in the relative weight of these between subjects, and particularly between professions. It should be noted, for example, that the housekeepers use much less variety of grasps than the machinists. For the housekeepers, the first five grasps already provide 80 percent of the grasping duration, while for the machinists 10 or 11 grasps are required to reach a similar level. Fifteen housekeeper grasps supply 95 percent of the grasping duration, while 19 grasps are required for the machinists.

In the following sections, we discuss grasp use by profession, after which we present a brief discussion of each of the top 10 grasps, identifying important characteristics, and reasoning for the uses of each. Note that common objects associated with the grasps will be mentioned. Due to the high number of objects encountered in the data set (441 in total based on the initial object description by the raters), rigorous quantitative object analysis is challenging and beyond the scope of this work; however, some of the general qualitative patterns will still be discussed.

\subsection{Housekeeper Grasp Use}

In general, the housekeeper data were more dominated by a limited number of grasps and by a set of common cleaning objects, such as towels, mops, and spray bottles. This is important to keep in mind when interpreting the grasp statistics which are dominated by the housekeeper data. The housekeepers performed many cleaning and tidying tasks during their work, such as rearranging objects, wiping surfaces, mopping, and folding.

The housekeepers utilized a high percentage of power grasps $(55,51$ percent). Much of the precision disk data involved using a towel to wipe a surface, and might be better described as some form of power grasp. Thus, the true proportion of power grasps for the housekeepers could be even higher. This indicates that they are doing relatively little dexterous, within-hand manipulation, which generally must be done using precision grasps [23]. This is to be expected, because most of the cleaning tasks observed, such as scrubbing with towels or cleaning with a vacuum, require only that the cleaning tool is fixed to the arm-within hand motion is generally unnecessary.

Both housekeepers used the medium wrap and precision disk grasp much more frequently than any other grasp, and with a greater frequency than the machinists. The medium wrap was frequently used with spray bottles and mops, while the precision disk was used with towels. Housekeeper 1 utilized the power sphere and index finger extension grasps much more than the other subjects, partially due to her personal method of grasping soft rags for washing (power sphere) and particular means of holding a broom (index finger extension). Housekeeper 2 utilized the extension type and parallel extension grasps more than any other subject, in part, due to frequent usage of these grasps to handle the sponge and towel objects.

\subsection{Machinist Grasp Use}

The machinist data involved tasks such as using large tools (e.g., milling machine, lathe, table saw), small tools (e.g., calipers, files), and miscellaneous other tasks such as writing. Unlike the housekeepers, the machinists use a much wider set of grasps, and interact with a larger number of objects. The machinists use precision and intermediate grasps more extensively than the housekeeper subjects. For example, lateral pinch, tripod, and lateral tripod are heavily used by both machinists for tasks such as manipulating machine knobs and small work pieces. While the machinists do use significantly fewer power grasps than the housekeepers, some power grasps are still important for machining work. Two examples are the index finger extension, used often with files and hammers, and the medium wrap, used frequently with pliers and wrenches.

\subsection{Specific Grasp Characteristics}

The characteristics of the top 10 grasps (by even weighting of the frequency and duration proportion for the entire data set) will now be discussed briefly, in terms of criteria such as frequency, objects handled, duration histograms, and common grasp transitions:

1. Medium wrap is heavily weighted by the housekeepers, and, in general, it is frequently used with larger cylindrical objects. It is commonly used for both initial object pickup and release, and has quite a few longer duration instances in the duration histogram tail. This suggests that the grasp is well suited to longer duration activities such as carrying objects. Common transitions occur with the index finger extension grasp, emphasizing a close relationship between these two grasps.

2. Precision disk is primarily so high in the ranking due to its frequent use to classify wiping motions using towels in the housekeeper data set. In the case of these frequent towel grasps, the precision description and hand configuration are somewhat inaccurate. For these towel grasps, it might have been more accurate for the raters to use a palmar grasp to express the hand pressing the towel against a surface, or a power sphere when the hand is wrapped around a bunched towel. In addition to the dominant towel data, it is also used with many other compliant objects, and with some small handles and other miscellaneous objects. The grasp is used for a very wide variety of durations. Precision disk most frequently transitions to and from power sphere, and also frequently follows lateral pinch. It is only very rarely used to initially pick up or to release objects, and is, thus, likely used mainly for manipulation after the object is already grasped.

3. Lateral pinch is used with smaller knobs, flat objects such as keys (it has been called a "key pinch"), and cords. It is used less for part handling than many grasps in the machinist data set. This grasp has a limited tail in the duration histogram-it is used almost exclusively in short durations. The most common grasp transitions occur to and from precision disk, and lateral pinch is used frequently for initial prehension and object release. This suggests that lateral pinch may frequently be used to pick up an object for precision disk manipulation. Given the heavy weighting of the towel object for the precision disk grasp, this suggests that a lateral pinch was often used to initially pick up 
towels, which were then manipulated in other grasps. Beyond object effects, lateral pinch does have particular properties that make it unique and useful. It is a strong grasp that functions along an orthogonal grasp axis compared with grasps such as medium wrap. It may also help to place the object in a position where other parts of the hand do not get in the way, and where wrist rotation is easy.

4. Tripod is used frequently for small knobs and small parts, as well as other small objects such as screws, drill bits, end mills, and micrometers. The tripod grasp is used almost exclusively for short durations. It frequently transitions to and from the lateral tripod grasp, and occasionally to and from lateral pinch. The grasp is quite commonly used for initial prehension and object release and for small objects and fine manipulation. It is much more frequently used for initial prehension than the visually similar lateral tripod grasp.

5. Lateral tripod is frequently used for handling small parts and tools, especially those with a small cylindrical shape, such as writing utensils, end mills, and hex wrenches. In terms of object types, it appears to be similar to the lateral pinch. The duration histogram drops off sharply with very few long duration grasps. It is most connected to tripod, thumb-2 finger, and light tool through grasp transitions. It is very rarely used for initial object prehension and release. Overall, the lateral tripod appears to be ill suited for grabbing objects, but is used for short duration manipulations especially of short, thin cylindrical objects.

6. Power sphere is heavily weighted by use with the towel in the housekeeper data. However, it appears, in general, to be used with many other soft, compliant objects, such as pillows, sponges, stuffed animals, as well as with some types of machine handles. It is frequently used for short durations, but also has several instances of longer duration grasps. It is used less frequently than average for initial prehension and for object release, which is somewhat surprising. By far the most frequent transitions are to and from precision disk, with occasional lateral pinch transitions. This suggests that precision disk and lateral pinch may be used to initially pick up objects that are later held in a power sphere grasp. Again, this particular transition sequence may apply mainly for towels or similarly compliant objects due to the heavy towel object weighting on these grasps.

7. Thumb-2 finger is frequently used with small parts and tools, especially small and flat or cylindrical tools and objects. It is also used with a few miscellaneous other objects such as towels and blankets. It has a fair amount of longer duration grasps. The most common transitions occur to and from the lateral tripod, transitioning to and from lateral pinch is also common. It is commonly used for object pickup and fairly often for object release. Overall, the thumb-2 finger grasp appears to be well suited to small and flat or cylindrical objects, and has a close connection with the lateral tripod and lateral pinch grasps, which likely reflects the fairly simple changes in hand configuration required to make these grasp transitions.

8. Index finger extension is used frequently with cylindrical objects and tools, especially those that require larger forces. Some object examples include the mop, file, vacuum, aerosol can, hammer, and toilet brush. Along with the precision disk grasp, index finger extension shows one of the highest proportions of long duration grasps. It most frequently transitions from medium wrap, or slightly less often to medium wrap, and has very few other transitions. Compared to medium wrap, the different index finger position may provide added control and a better object angle for strong opposition between the finger pads and the skin overlying the thenar eminence. The index finger extension grasp may also add to the sensitivity of the hand in reacting to changing tool forces. Overall, the index finger extension grasp appears particularly well suited to applying large and controlled forces with tools. However, the grasp is very rarely used for initial object pickup or final object release, probably because the extended index finger has little utility during these phases of manipulation.

9. Light tool is used with various cylindrical objects such as calipers, wrenches, machine handles, and vacuum hose ends. An average amount of longer duration grasps are observed. Light tool commonly transitions to and from the lateral tripod, or sometimes thumb-2 finger. Light tool is used quite frequently for initial object prehension. This is probably a similar prehension motion to the thumb-2 finger grasp, which is also quite commonly used to initially grasp objects. Perhaps grasping with the thumb extended in the light tool configuration is particularly convenient for objects that are not resting completely on a surface, such as the machine handle or vacuum hose end. In these cases, the thumb would be out of the way during a relatively easy grasp, while in other cases the thumb might need to be more active during the initial prehension phase.

10. Thumb-3 finger is used most for manipulation of small parts and short, rod-shaped objects. The amount of long duration grasps is a bit above average. Transitions to and from lateral pinch are most common, with lateral tripod and tripod transitions also being fairly common. This suggests that the particular motion of flipping an object back and forth between the thumb-3 finger configuration and lateral pinch configuration may be important in manipulating small objects, especially short, thin rods.

\section{Conclusions AND Future Work}

Due to the huge variety of manipulation tasks done with the hands, it is difficult to draw any absolute truth from even such a large data set. There is a large amount of variability between subjects, and even more between professions. This is both due to the differences in the tasks performed, as well as the individual preferences and manipulation strategies of each subject. However, despite these differences, there are a number of things that can be learned from the results presented here.

Different types of work vary hugely in terms of the variety of grasps and objects interacted with. The housekeepers used relatively few grasps most of the time, and interacted with relatively few objects for a large proportion of the study-mainly interacting with the cleaning supplies needed to carry out their work. The machinists, on the other hand, interacted with a much broader spread of objects and used a wide variety of the grasps. As a result, the grasps dominated by the machinist data give us general characteristics of the grasps, while the housekeeper dominated 
grasps tell us more about certain aspects of the grasps, such as the handling of compliant objects. One strength of the housekeeper data set is that, while heavily weighted by a few cleaning objects, the data do still contain quite a few household objects that were moved around during the cleaning process. This data may be useful to further analyze what grasps are used to transport household objects, but this analysis would require careful and systematic masking of the many cleaning objects in the data set, since the highfrequency cleaning objects are probably not as important in household object transport during standard activities of daily living.

By looking at each grasp from the multiple perspectives of frequency, objects handled, duration histograms, and grasp transitions, it is possible to obtain a better overall picture of how each grasp is used. For example, index finger extension and medium wrap were shown to frequently trade off with one another (transition data), but index finger extension seems particularly well suited to tools that require both high force and some precision (object data), while medium wrap is used more for initial prehension (transition data). Both grasps have long mean durations (duration data), suggesting they may be used more for longer term object transport rather than quick dexterous movements.

Several extensions are possible for the present work. We plan to classify the object types being grasped, as well as the task being performed. We then plan to investigate relationships between different grasps and object types, examine the frequency of general classes of tasks, and analyze how different subjects perform similar tasks. Of course, the current study could also be extended by simply adding subjects and professions.

The present study has highlighted that future work could be done to improve grasp taxonomies. For example, the prevalence of the towel object in the housekeeper data set has highlighted the need for a better way to classify the grasps of compliant objects. Most of the existing taxonomies appear to have been designed with certain geometries of rigid objects in mind, so attempting to accurately classify soft objects with the same system is difficult. Confusion matrix methods could be used to help develop a set of grasp types for compliant objects, which raters can reliably distinguish.

We believe that this work, while subject to some limitations, provides valuable information about how different grasps are actually used in a real-world setting. Humans use their hands in a wide variety of ways, such that the full picture cannot possibly be captured in one study. The work presented in this paper seeks to capture a small portion of that picture, and in particular how frequently different prehensile grasps are used in unstructured "real-world" environments. While even that aspect was not fully captured here as we studied only four subjects from two professions, we believe many of the results are representative of general hand use, such as the relative proportion of power, intermediate, and precision grasps, as well the trends on grasp duration, for instance. We hope that the information provided will be of use in many fields, such as designing robotic and prosthetic hands with reduced mechanical complexity or developing rehabilitation techniques to prioritize various aspects of human hand function.

\section{ACKNOWLEDGMENTS}

The authors would like to thank Kayla Matheus for her help in creating the hand illustrations used in this paper, and Thomas Feix for a few helpful discussions relating to the paper content. This work was supported in part by the National Science Foundation, grant IIS-0952856. A preliminary version of this paper was presented at the 2011 IEEE International Conference on Robotics and Automation [18].

\section{REFERENCES}

[1] J. Napier, "The Prehensile Movements of the Human Hand," J. Bone Joint Surgery, vol. 38B, no. 4, pp. 902-913, 1956.

[2] G. Schlesinger, "Der Mechanische Aufbau Der Kunstlichen Glieder," Ersatzglieder und Arbeitshilfen für Kriegsbeschädigte und Unfallverletzte, pp. 321-661, Verlag von Julius Springer, 1919.

[3] L.M.K. Boelter, A.D. Keller, C.D. Taylor, and V. Zahm, Studies to Determine the Functional Requirements for Hand and Arm Prosthesis. Dept. of Eng., Univ. California, 1947.

[4] C.L. Mackenzie and T. Iberall, The Grasping Hand, pp. 3-14. NorthHolland, 1994.

[5] M. Santello, M. Flanders, and J.F. Soechting, "Postural Hand Synergies for Tool Use," J. Neuroscience, vol. 18, no. 23, pp. 1010515, Dec. 1998.

[6] L. Sperling and C. Jacobson-Sollerman, "The Grip Pattern of the Healthy Hand during Eating," Scandinavian J. Rehabilitation Medicine, vol. 9, no. 3, pp. 115-121, 1977.

[7] M.R. Cutkosky, "On Grasp Choice, Grasp Models, and the Design of Hands for Manufacturing Tasks," IEEE Trans. Robotics and Automation, vol. 5, no. 3, pp. 269-279, June 1989.

[8] C.C. Kemp, "A Wearable System That Learns a Kinematic Model and Finds Structure in Everyday Manipulation by Using Absolute Orientation Sensors and a Camera," PhD thesis, Massachusetts Inst. of Technology, 2005.

[9] Hosmer, "Hooks," http://hosmer.com/products/hooks / index.html, Aug. 2012.

[10] A.M. Dollar and R.D. Howe, "The Highly Adaptive SDM Hand: Design and Performance Evaluation," Int'l J. Robotics Research, vol. 29, no. 5, pp. 585-597, Feb. 2010.

[11] N. Ulrich and V. Kumar, "Grasping Using Fingers with Coupled Joints," Proc. ASME Mechanisms Conf., 1988.

[12] S. Hirose, "The Development of Soft Gripper for the Versatile Robot Hand," Mechanism and Machine Theory, vol. 13, no. 1, pp. 351-359, 1978.

[13] L. Birglen, C. Gosselin, and T. Laliberté, Underactuated Robotic Hands, vol. 40. Springer Verlag, 2008.

[14] M.T. Mason, A. Rodriguez, S.S. Srinivasa, and A.S. Vazquez, "Autonomous Manipulation with a General-Purpose Simple Hand," Int'l J. Robotics Research, vol. 31, no. 5, pp. 688-703, Dec. 2011.

[15] T. Bionics, "I-Limb Ultra," http://www.touchbionics.com/ products/active-prostheses/i-limb-ultra/, Aug. 2012.

[16] Ottobock, "Ottobock - System Electric Hands," http://www. ottobock.com/cps/rde/xchg/ob_com_en/hs.xsl/5445.html, Aug. 2012.

[17] T. Feix, R. Pawlik, H. Schmiedmayer, J. Romero, and D. Kragic, "A Comprehensive Grasp Taxonomy," Proc. Robotics, Science and Systems Conf:: Workshop Understanding the Human Hand for Advancing Robotic Manipulation, 2009.

[18] J. Zheng, S. De, L. Rosa, and A. Dollar, "An Investigation of Grasp Type and Frequency in Daily Household and Machine Shop Tasks," Proc. Int'l Conf. Robotics and Automation, pp. 4169-4175, 2011.

[19] Y. Matsumoto, Y. Nishida, Y. Motomura, and Y. Okawa, "A Concept of Needs-Oriented Design and Evaluation of Assistive Robots Based on ICF," Proc. IEEE Int'l Conf. Rehabilitation Robotics, 2011.

[20] J. Cohen, "A Coefficient of Agreement for Nominal Scales," Educational and Psychological Measurement, vol. 20, no. 1, pp. 37-46, Apr. 1960.

[21] A.J. Conger, "Kappa Reliabilities for Continuous Behaviors and Events," Educational and Psychological Measurement, vol. 45, no. 4, pp. 861-868, Dec. 1985. 
[22] T.C. Hesterberg, D.S. Moore, S. Monaghan, A. Clipson, and R. Epstein, "Bootstrap Methods and Permutation Tests," Introduction to the Practice of Statistics, W.H. Freeman, 2005.

[23] I.M. Bullock and A.M. Dollar, "Classifying Human Manipulation Behavior," Proc. IEEE Int'l Conf. Rehabilitation Robotics, 2011.

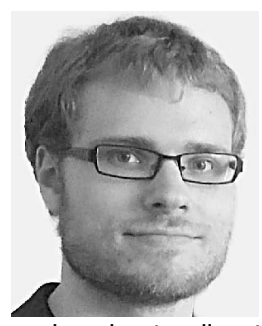

Ian M. Bullock received the BS degree in engineering from Harvey Mudd College, Claremont, California, in 2010. He has been working toward the PhD degree at Yale University since the fall of 2010. His work experience has ranged from programming educational neuroscience modules to designing circuits for a particle accelerator. His research interests include human dexterity and robotic manipulation, with particular emphasis on using and understanding the capabilities of the human hand to improve the design of robotic and prosthetic manipulators. $\mathrm{He}$ is a student member of the IEEE.

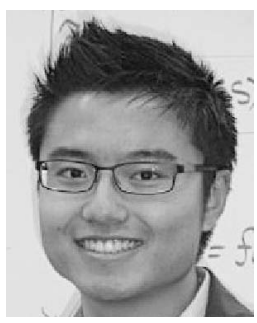

Joshua Z. Zheng received the BS degree in biomedical engineering from the Johns Hopkins University in 2009, and the MS degree in mechanical engineering from Yale University in 2011. His concentration at Johns Hopkins was bioinstrumentation, and he also studied robotics, with a focus in human grasping and manipulation. He interned with medical device companies such as Boston Scientific, Medtronic, and St. Jude Medical, working on pacemakers and drug delivery devices. He is currently with at the Institute for Physical Sciences (IPS) doing software development and machine learning.

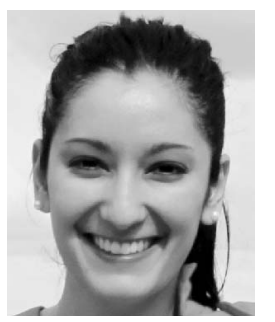

Sara De La Rosa is working toward the BS degree in mechanical engineering, and is as a research assistant in the Yale GRAB Lab. During the summer of 2010, she was named a Science, Technology and Research Scholar as a participant in the Yale STARS Summer Research Program, which supports historically underrespresented students in the sciences, engineering, and mathematics. She was a part of an interdisciplinary team on a design project for smallholder farmers in sub-Saharan Africa in the inaugural Appropriate Technology for the Developing World course at Yale during the spring of 2011.

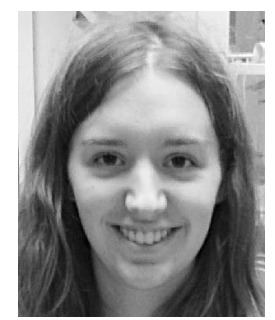

Charlotte Guertler is working toward the BS degree in mechanical engineering at Yale University. She has been involved with many research projects ranging from fluid mechanics in microgravity with NASA to data collection and analysis of human grasping and manipulation.

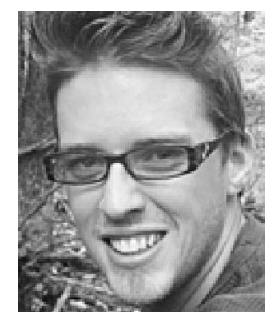

Aaron M. Dollar received the BS degree in mechanical engineering from the University of Massachusetts at Amherst, the SM and PhD degrees in engineering sciences from Harvard University, and conducted two years of postdoctoral research at the MIT Media Lab. He has been an assistant professor of mechanical engineering and materials science at Yale University since 2009. His research interests include human and robotic grasping and dexterous manipulation, mechanisms and machine design, and assistive and rehabilitation devices including upper-limb prosthetics and lower-limb orthoses. He is a cofounder and editor of RoboticsCourseWare.org, an open repository for robotics pedagogical materials, and the recipient of the 2011 Young Investigator Award from the US Air Force Office of Scientific Research, the 2010 Technology Review TR35 Young Innovator Award, and the 2010 US National Science Foundation CAREER Award. He is a member of the IEEE.

$\triangleright$ For more information on this or any other computing topic, please visit our Digital Library at www.computer.org/publications/dlib. 